\title{
Covid-19, globalización,
} complejidad e incertidumbre: algunas reflexiones sobre gestión empresarial en tiempos de crisis y más allá

\section{Covid-19, globalization, complexity and uncertainty: some reflections on business management in crisis times and beyond}

Arturo Rodríguez-Castellanos ${ }^{1}$, Nerea San-Martín-Albizuri²

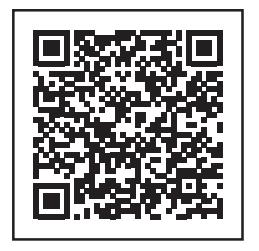

Palabras clave:

Covid-19, crisis económica, globalización, complejidad, incertidumbre, respuesta empresarial

Artículo de reflexión

Fecha de recepción: 01/06/2020

Fecha de aceptación: 30/06/2020

Esta publicación se encuentra bajo licencia:

Creative Commons ReconocimientoNoComercialSinObraDerivada 4.0 Internacional

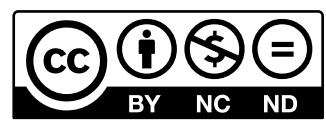

\section{Resumen}

La pandemia de la covid-19 ha inducido una fuerte crisis económica, de duración e intensidad difíciles de prever. Muchas empresas de diferentes sectores han padecido sus efectos.

¿Qué puede hacerse para asegurar la supervivencia de las empresas, no solo frente a los efectos inmediatos de la crisis, sino, a más largo plazo, para superar las dificultades de un entorno progresivamente complejo e incierto? En este trabajo pretendemos aportar algunas respuestas al respecto.

Por ello, tras examinar las características de esta crisis, tanto en sus diferencias como en sus similitudes con crisis anteriores, y aventurar algunas de las consecuencias esperables de la misma, se abordan

1 Doctor en Ciencias Económicas y Empresariales, Cátedra de Empresa Familiar, Universidad del País Vasco UPV/EHU, y Real Academia de Ciencias Económicas y Financieras, España, arturo.rodriguez@ehu.eus, Código ORCID: https://orcid.org/0000-0002-9436-423X

2 Doctora en Ciencias Económicas y Empresariales, Facultad de Economía y Empresa, Universidad del País Vasco UPV/EHU, España, nerea.sanmartin@ ehu.eus, Código ORCID: https://orcid.org/0000-0001-7714-4182 


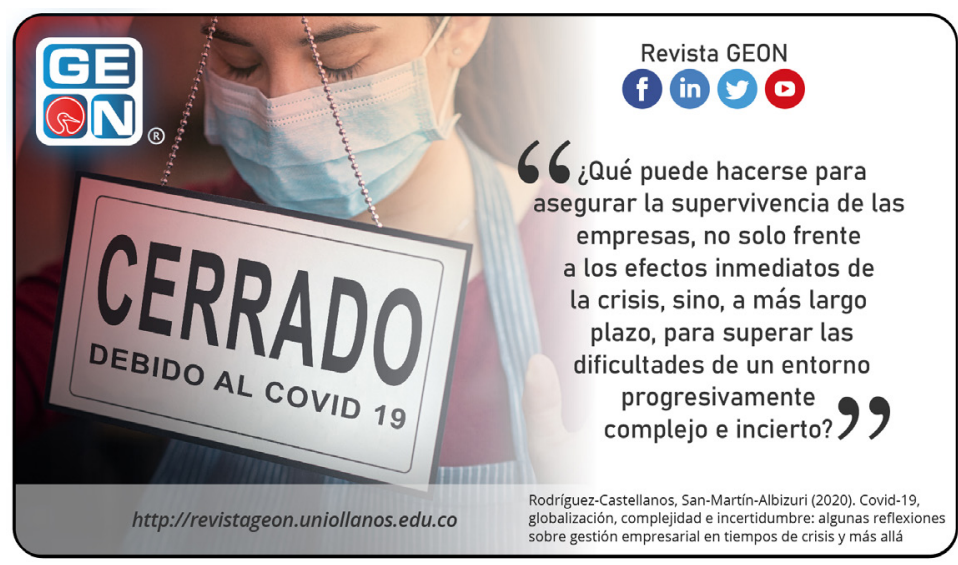

sugerencias en el ámbito empresarial, tanto para superar sus efectos inmediatos como para afrontar la supervivencia empresarial a más largo plazo. A este último respecto, se plantea la consecución de dos propósitos básicos: la innovación y la sostenibilidad. Y para ello deben desarrollarse una serie de capacidades, en especial tres ambidestrezas.

Palabras claves: Covid-19, crisis económica, globalización, complejidad, incertidumbre, respuesta empresarial

\section{Abstract}

The covid-19 pandemic has induced a strong economic crisis, whose duration and strength are difficult to predict. Many companies in different sectors have suffered its effects.

What could be done to ensure the survival of firms, not only to cope with the immediate effects of the crisis, but to overcome the difficulties of an increasingly complex and uncertain environment?. The aim of this paper is to provide some answers on

Cómo citar este artículo / Toreference this article:

Rodríguez-Castellanos, A., \& SanMartin-Albizuri, N. (2020). Covid-19, globalización, complejidad e incertidumbre: algunas reflexiones sobre gestión empresarial en tiempos de crisis y más allá. Revista GEON (Gestión, Organizaciones Y Negocios), 7(2), 1-17. https://doi. org/10.22579/23463910.219 this point.

Thus, we examine the characteristics of this crisis, considering both the differences and the similarities with previous crises, and outlining some of the expected consequences of it. In addition, we address suggestions in the business field, both to overcome its immediate effects and to face longer-term 
Cómo citar este artículo /

Toreference this article:

Rodríguez-Castellanos, A., \& SanMartin-Albizuri, N. (2020). Covid-19, globalización, complejidad e incertidumbre: algunas reflexiones sobre gestión empresarial en tiempos de crisis y más allá. Revista GEON (Gestión, Organizaciones Y Negocios), 7(2), 1-17. https://doi. $\operatorname{org} / 10.22579 / 23463910.219$ business survival. Regarding this last point, it is proposed the achievement of two basic purposes: innovation and sustainability. And for this, a number of skills must be developed, especially three ambidexterities.

Keywords: Covid-19, economic crisis, globalization, complexity, uncertainty, business response

Códigos JEL: F440, F610, M10

\section{Introducción}

La pandemia de la covid-19 no solo ha provocado una crisis sanitaria mundial sin precedentes en muchas décadas, sino que ha inducido una crisis económica cuya duración e intensidad son desconocidas, pero que pueden ser muy amplias. Bien por el lado de la oferta, bien por el de la demanda, ha afectado a empresas de muy diversos sectores de actividad.

Ante estas circunstancias, muchos dirigentes empresariales se plantean la supervivencia futura de sus organizaciones, y cómo deben guiar su acción para conseguirla. Por ello, la finalidad del presente trabajo es proporcionar algunas reflexiones sobre la crisis actual -sus características como derivada de la complejidad y sus repercusiones económicas y empresariales- y sobre la actuación de las empresas ante ella. Pero, más allá, también pretende proporcionar sugerencias sobre la gestión en un entorno cada vez más complejo e incierto.

Por ello, tras este apartado introductorio, en el siguiente se analizan las características de la crisis económica actual, destacando sus importantes diferencias con las acaecidas en las últimas décadas, pero también algunas semejanzas, como su vinculación a la complejidad debida en gran parte a la globalización. Posteriormente, se aventuran sus posibles consecuencias desde el punto de vista económico, pero principalmente desde el empresarial.

En vista de tales consecuencias, en el cuarto apartado se dan una serie de indicaciones al objeto de proporcionar a directivos y empresarios ideas útiles para orientarse tanto en la coyuntura más inmediata como en el entorno futuro que se vislumbra.

En quinto lugar se presentan las conclusiones de trabajo, finalizando con las referencias bibliográficas.

\section{Una crisis como ninguna otra}

El título de este apartado podría ser considerado como una afirmación trivial. En efecto, todas las crisis económicas son diferentes, cada una de ellas es única. Pero si lo anterior es cierto, también lo es que algunas cri- 
Covid-19, globalización, complejidad e incertidumbre: algunas reflexiones sobre gestión empresarial en tiempos de crisis y más allá

sis son "más diferentes" que otras. Y tal vez la "crisis de la covid-19" sea la "más diferente" en mucho tiempo.

Veamos por qué. Así, la actual crisis:

- No tiene un origen financiero, como sucedió en las crisis asiáticas de 1997-1998, la crisis de las ".com" del año 2000, o la "crisis subprime" de 2008.

- Tampoco tiene su origen en desequilibrios de variables económicas, como la "crisis del petróleo" de los años setenta del pasado siglo, la crisis japonesa de 1989-1990, la mexicana de 1994, o la argentina de 2001.

- Tiene su origen en un shock externo, en este caso de carácter sanitario. Pero no ha incidido en una frágil situación económico-financiera previa, pues antes del estallido de la pandemia la economía mundial estaba en claro crecimiento.

Ahora bien, ¿tiene algo en común esta crisis con otras anteriores? En nuestra opinión sí, al menos con las que hemos denominado "crisis de la globalización" (Rodríguez-Castellanos, Urionabarrrenetxea-Zabalandikoetxea y San-Martín-Albizuri, 2008), o "crisis de la complejidad" (Rodríguez-Castellanos, 2019a). En efecto, consideramos que la complejidad debida a la globalización ha sido uno de los desencadenantes, tanto de la crisis sanitaria, como de la crisis económica subsiguiente.

La globalización es un fenómeno que lleva décadas aumentando la inter- conexión en múltiples niveles, lo cual origina incrementos de complejidad, que puede conllevar efectos positivos -"círculos virtuosos", emergencia de nuevas estructuras favorables (Prigogine y Stengers, 1984, MitcheII, 2009)- pero también negativos, como "efectos mariposa" o "efectos en cascada" (Innerarity, 2020), "cisnes negros" -sucesos de muy baja probabilidad y de gran impacto- (Taleb, 2007), procesos "caóticos" (Prigogine y Stengers, 1984), "contagios" (Mendoza y Quadrini, 2010), etc. Todo ello da lugar a incertidumbre e imprevisibilidad.

Visto lo anterior, parece claro que globalización y complejidad están en la base de esta crisis: sin la compleja interconexión física y humana debida a la globalización, no habría sido posible la expansión vertiginosa del SARS-CoV-2, y sin las fuertes interconexiones económicas a través de cadenas de suministros globales no se habría iniciado la crisis económica de forma tan fuerte, ni se habría producido el rápido "contagio" de la misma, aspecto por otra parte compartido con muchas crisis anteriores (Rodríguez-Castellanos, 2019a).

Continuando con la evolución de la crisis, puede comprobarse el "efecto en cascada" ya mencionado, como se verá a continuación (Fariza, 2020; Nolte, 2020).

La crisis al principio ha sido "de oferta", debido a la ruptura de las cadenas de suministros de muchas grandes empresas multinacionales, que han ido dependiendo cada vez más de 
instalaciones chinas, con suministros just in time (Bofinger et al., 2020). Además, las instalaciones más importantes del mundo para la fabricación de equipos de protección sanitaria individual se encuentran concentradas en China y en algunos otros países emergentes, con lo que el dramático incremento en la demanda mundial de estos equipos no ha podido ser debidamente cubierto. Esta crisis de oferta se ha ido transmitiendo a otros países, en la medida en que las normas de confinamiento han impedido la elaboración de ciertos bienes o la prestación de ciertos servicios

Pero la crisis también se ha extendido hacia la demanda. La paralización de actividades y las medidas de distanciamiento social han originado descensos en el consumo superiores a los registrados en la crisis de 2008. Los sectores más afectados, ahora y en el futuro, están siendo el comercio presencial, la hostelería, la restauración, el turismo, el transporte de viajeros y los espectáculos (Lucchese y Pianta, 2020). A esto se añaden los efectos económicos adversos provocados por la pérdida de empleos y la caída de ingresos, actual o esperada. Una de las consecuencias más dramáticas al respecto ha sido la caída en el precio del petróleo (Belinchón, 2020a, 2020b).

Y también ha sido una crisis bursátil. Simultáneamente a lo anterior, las bolsas más importantes del mundo sufrieron espectaculares descensos (Zhang, Hu y Ji, 2020), pronto atemperados por la decisión de los principales bancos centrales de sumi- nistrar liquidez prácticamente ilimitada (Adrian y Natalucci, 2020; Ramelli y Wagner, 2020).

¿Originará todo ello una crisis bancaria? Ciertamente, como consecuencia de las medidas adoptadas ante la "crisis subprime", en general la banca en los principales países se encuentra saneada y capitalizada (Lee, 2020). Ahora bien, teniendo en cuenta que en las actuales circunstancias las consecuencias económicas de la pandemia son imposibles de prever, no puede excluirse un escenario de crisis en el sector debida al exceso de morosidad (Adrian y Natalucci, 2020, Beck, 2020).

Muy preocupante asimismo es la posibilidad de una "crisis de deuda", en la medida en que el endeudamiento público necesario para cubrir los gastos y apoyos de todo tipo, exigidos por la lucha contra la epidemia, llegue a ser insoportable (Euromoney, 2020a, 2020b). Tal vez el mayor problema se esté gestando en ciertos países en desarrollo y emergentes, que ya estaban en una situación de deuda externa difícilmente sostenible y que están padeciendo la mayor huida de capitales registrada hasta el presente (Adrian y Natalucci, 2020; Reinhart y Rogoff, 2020).

Otra característica de esta crisis, ya mencionada, también consecuencia de la complejidad, ha sido la imprevisibilidad. Podemos decir incluso que es un ejemplo perfecto de "cisne negro". Las autoridades, en general, estaban escasamente preparadas para anticipar el enorme nivel de ex- 
Cavid-I9, globalización, complejidad e incertidumbre: algunas reflexiones sobre gestión empresarial en tiempos de crisis y más allá

tensión global que iba a alcanzar un patógeno, que, aun con una baja tasa de mortalidad, ha sido capaz de hacer colapsar sistemas sanitarios a priori suficientemente satisfactorios. Y tampoco para anticipar sus tremendas repercusiones económicas.

Ahora bien, la imprevisibilidad no es una característica nueva, pues ya se manifestó claramente en la "crisis subprime", y en general en las ocurridas en las tres últimas décadas. De hecho, análisis efectuados al objeto de contrastar la capacidad de ciertos instrumentos -índices de riesgo país, ratings país- para anticipar tales crisis han arrojado resultados negativos (Reinhart, 2002; San-Martín-Albizuri y Rodríguez-Castellanos, 2011, 2015).

Visto esto, parece que tanto el mundo actual, como el futuro vislumbrable, serán complejos e inciertos. Deberíamos, por tanto, asumir una mayor posibilidad de acontecimientos imprevisibles y de grandes repercusiones.

Pasamos a continuación a considerar con más detenimiento las posibles consecuencias de esta crisis para la economía y para las empresas.

\section{Consecuencias}

Los efectos de esta crisis sobre la economía del mundo son prácticamente imposibles de prever, dada la incertidumbre sobre la evolución de la pandemia en los diferentes países. Ahora bien, diversas entidades y organismos internacionales han elaborado estimaciones, que en todo caso anticipan una caída del PIB mundial. Es, por tanto, esperable un fuerte impacto negativo sobre la economía, tal vez con destrucción de empleo más que proporcional, ya que algunos de los sectores más afectados -comercio, hostelería, restauración, turismoson intensivos en mano de obra. El efecto por países será muy posiblemente asimétrico, según la importancia relativa de estos últimos sectores (Mann, 2020). Pero más allá de la recesión económica, hay aspectos en el mundo económico y empresarial que muy posiblemente experimentarán importantes cambios.

Nos referiremos en primer lugar a la alta posibilidad de que las autoridades de muchos países tomen decisiones para evitar que una futura pandemia vuelva a encontrar desprotegidos a sus sistemas sanitarios (Boone et al., 2020). Considerando que la atención sanitaria es un asunto estratégico, procurarán evitar que la provisión de activos sensibles -como instrumentos de protección individual, medicinas y otros- dependa exclusivamente del exterior. Por tanto, es esperable un apoyo público a empresas locales productoras de estos activos, y también a empresas que, aunque no los produzcan habitualmente, estén en condiciones de transformarse en poco tiempo para poder producirlos. Es también esperable un mayor apoyo a la sanidad y a la investigación sanitaria. En este sentido, se está abogando por potenciar el estado de bienestar, para que vaya más allá de la prestación de servicios de salud, y se logre implantar un modelo de desarrollo más sostenible y de mejor calidad (Lucchese y Pianta, 2020). 
Otra consecuencia de esta crisis que parece evidente es el desarrollo futuro de las tecnologías de la información y de la comunicación (TIC), y su empleo tanto en el ámbito individual y educativo, como en el organizacional.

Otro posible cambio radicará en la reestructuración de las cadenas globales de suministro de muchas industrias, para no hacerlas depender casi exclusivamente de un solo país -como China- (Browne, 2020). Esto implicará, por una parte, una mayor diversificación de proveedores, y por otra, la "re-localización" o "retorno de producción", esto es, la vuelta a la fabricación en entornos próximos al consumo (Fanjul, 2020). Ahora bien, este no es un fenómeno nuevo, pues la tendencia a desplazar la producción de China a otros países emergentes ya existía previamente, debido al alza de los costes laborales y al endurecimiento en las regulaciones medioambientales. Por otra parte, la "re-localización" también venía produciéndose, propiciada por varios factores (Boz y Tesar, 2018; HaIlward-Driemeier y Nayyar, 2018): i) el desarrollo tecnológico, que ha permitido reducir los costos de producción en muchas industrias; ii) la presión social y las normativas para reducir la "huella de carbono" han obligado en muchos casos a "internalizar" una parte de los costos de trasporte a larga distancia; iii) la tendencia a ajustarse a las necesidades concretas de la clientela mediante un producción próxima a la misma; iv) en el caso de las empresas estadounidenses, la guerra comercial con China. Pode- mos ver, por tanto, que a este respecto la actual crisis no va a cambiar unas tendencias ya existentes, pero claramente las agudizará.

Especialmente preocupante es el posible efecto negativo sobre las micro, pequeñas y medianas empresas, pues predominan en sectores especialmente afectados: comercio presencial, hostelería, restauración, etc. Son entidades con debilidades estructurales, que deberán ser objeto de especial atención por las autoridades, ya que responden de la mayor parte del empleo en casi todos los países. Por otra parte, también son más ágiles y flexibles, por lo que una política de apoyo inteligente a las mismas puede propiciar una rápida recuperación del empleo.

Y si de los diferentes sectores de actividad pasamos al ámbito macroeconómico, no debe dejar de preocuparnos, como ya se ha indicado, el efecto que sobre el endeudamiento público puedan tener las políticas adoptadas para combatir tanto la propia pandemia como sus efectos en la economía. A este respecto, los países en desarrollo y emergentes se encuentran en condiciones mucho peores. Además, el "retorno de producción" puede perjudicarles especialmente (Sahagún, 2020). En este sentido, para atajar una "crisis de deuda", deberá desarrollarse una gobernanza global con medidas audaces, incluyendo reestructuraciones y moratorias de la deuda externa.

Ahora bien, no parece que los desafíos que se presentan puedan ser 
afrontados con éxito sin el apoyo de la sociedad civil. En esta crisis hemos visto muchos y grandes ejemplos de organización espontánea de colectivos ciudadanos para abordar problemas tanto sanitarios como económicos y sociales. En este sentido, es esperable un mayor desarrollo de asociaciones, ONG y entidades del tercer sector, así como de iniciativas empresariales de responsabilidad social empresarial y de tipo colaborativo (Arellano Yanguas, 2020; Fontrodona y Muller, 2020)

\section{¿Qué hacer?}

¿Cómo debe adaptarse la gestión empresarial para afrontar la situación actual y la esperada en el futuro, según lo considerado en los apartados anteriores? En las siguientes páginas vamos a presentar una serie de indicaciones, con la finalidad no tanto de guiar la gestión empresarial, como de proporcionar ideas que esperamos sean útiles a directivos y empresarios para orientarse tanto en la coyuntura más inmediata como en el entorno futuro que se vislumbra.

Así, en la perspectiva más inmediata, esto es, los próximos meses en los que el impacto económico de la pandemia será previsiblemente más fuerte, estimamos que puede resultar una buena referencia el trabajo de Leiva y Guillén (2020), sintetizado en su "cuadrante de análisis estratégico para daños por la crisis" (tabla 1).

En él se consideran cuatro casos diferentes, según que la crisis haya originado, o no, daños en las operaciones de la empresa y/o daños a la clientela. $Y$ se proporcionan indicaciones para cada caso en cuatro campos de acción esenciales: Comunicación con la clientela, Operaciones, Flujos de tesorería y Gestión del talento humano.

A continuación se consideran cada uno de los casos:

1. Daños en las operaciones escasos, y clientela escasamente afectada: puede ser el caso del comercio de alimentación, o de la industria del software de telecomunicaciones (Figueras, 2020). Se puede seguir operando normalmente, pero debe efectuarse un seguimiento permanente de la evolución de la empresa, los clientes y los aliados.

2. Escasos daños en la clientela, pero importantes daños en las operaciones (e.g., por rotura de fuentes de aprovisionamiento): este puede ser el caso de la industria sanitaria y farmacéutica (Figueras, 2020). La prioridad será recuperar la normalidad cuanto antes, buscando fuentes de aprovisionamiento alternativas, o incluso considerando la posibilidad de elaborar o suministrar otros productos.

3. Daños en las operaciones escasos, pero fuertes dificultades en la clientela: empresas inmobiliarias, de transporte, de turismo o de comercio al por menor pueden encontrarse en esta situación (Figueras, 2020). Debe procurarse mantener el máximo de clientela, tal vez buscando nuevos canales de distribución, reconfigurando los 
productos o servicios u ofreciendo otros nuevos, y también buscar nueva clientela.

4. Fuertes daños en las operaciones, y también dificultades en la clientela: puede ser el caso de la industria de automoción (Figueras, 2020). Las circunstancias aconsejan una suspensión o cierre temporal, protegiendo las instalaciones y los activos claves.

Tabla 1. Cuadrante de análisis estratégico para daños por la crisis (próximos meses)

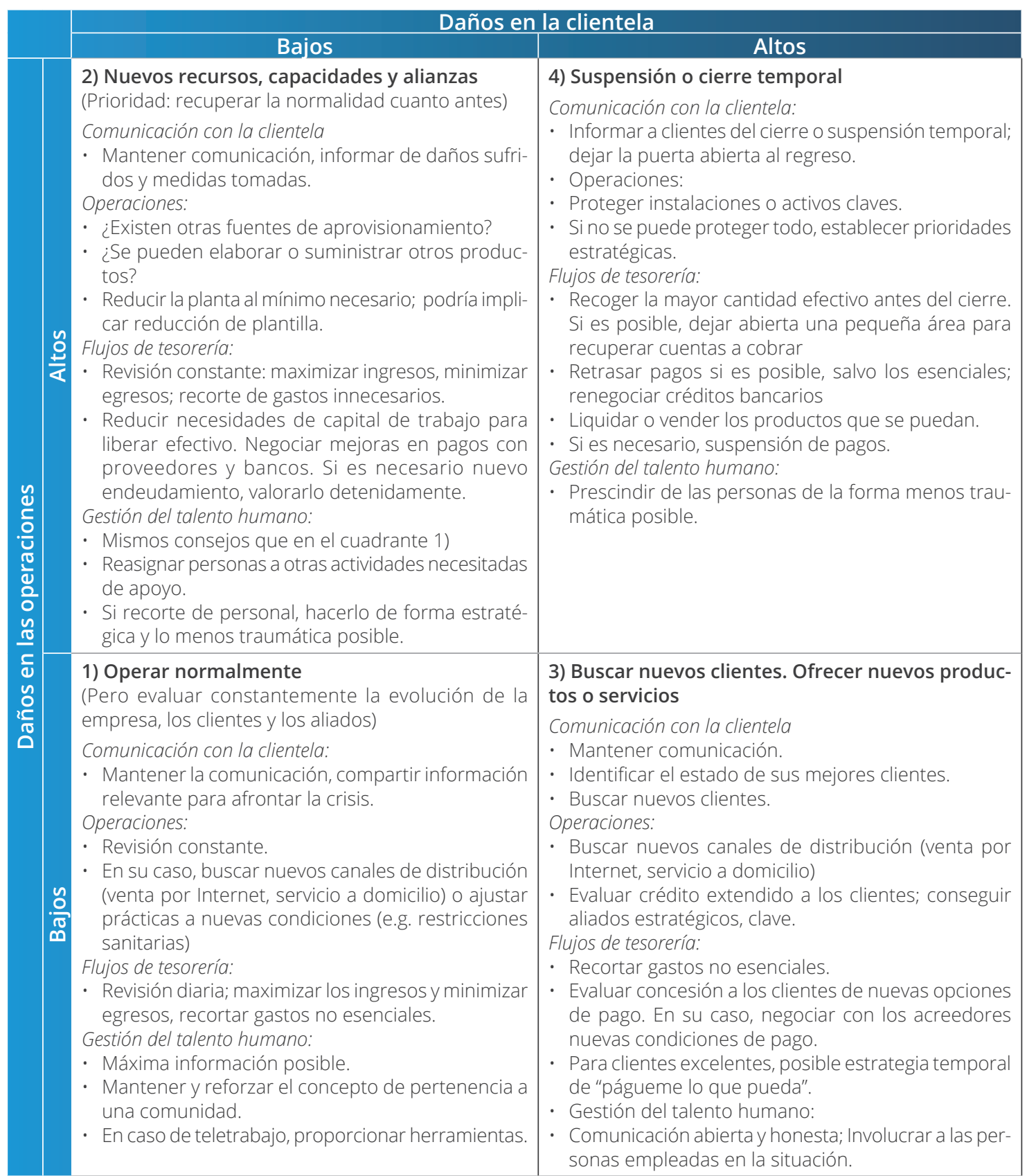

Fuente: elaboración propia sobre Leiva y Guillén (2020) 
Covid-19, globalización, complejidad e incertidumbre: algunas reflexiones sobre gestión empresarial en tiempos de crisis y más allá

Ahora bien, con una perspectiva más a largo plazo, y tras abordar el efecto inmediato de la crisis, hay que asumir que la empresa debe desenvolverse en un entorno cada vez más volátil, incierto, complejo y ambiguo (VUCA, por sus siglas en inglés: volatility, uncertainty, complexity, ambiguity) (Mack et al., 2016). Para afrontarlo, se debe pensar en términos de complejidad sistémica (Berger-Vachon et al., 2018; Innerarity, 2020), y asumir que la incertidumbre es una característica esencial del mundo, actual y futuro.

Pero la constatación de que nos movemos hacia un mundo más incierto no es necesariamente una mala noticia. De hecho, se están desarrollando mejores métodos para abordar decisiones en condiciones de incertidumbre (Gil-Aluja et al., 2015; Marchau et al., 2019). Por otra parte, la incertidumbre puede ser fuente de valor si se sabe aprovechar las oportunidades; así, no solo se deberá procurar la resiliencia ante acontecimientos no previstos, sino también buscar posiciones de "antifragilidad" (Taleb, 2012), que saquen partido de las nuevas circunstancias.

¿Y las personas? Entendemos que en el futuro su papel será más crítico todavía, pues llevarán a cabo las tareas más decisivas, que las tecnologías no serán capaces de efectuar. Y las empresas, por tanto, si quieren sobrevivir con éxito de forma sostenible en el nuevo entorno, deberán ser más humanas (De-Goñi-Oslé y Rodríguez-Castellanos, 2018; Rodríguez-Castellanos, 2019b).

Pero estas "nuevas personas para la nueva empresa" son diferentes, y en el futuro lo serán todavía más. En un mundo VUCA, las personas no buscarán únicamente una buena remuneración por su trabajo, sino también un propósito en sus vidas, sentirse parte de algo importante y necesario que beneficie al conjunto de la sociedad. Su motivación radicará cada vez más en el deseo de colaboración, la autonomía y el significado (Schwab, 2016). Únicamente las empresas que sean capaces de cumplir estas aspiraciones sobrevivirán en el futuro.

Teniendo en cuenta lo anterior, para que las empresas sean capaces de sobrevivir, adaptarse y crecer en este nuevo entorno, proponemos dos propósitos que deben guiar su actuación (Rodríguez-Castellanos, 2018, 2019b):

- Sostenibilidad: debe tenerse en cuenta que la empresa es un proyecto social, llevado a cabo conjuntamente por diversos grupos de interés -stakeholders-. Más allá de la mera sostenibilidad del negocio, debe buscarse también la sostenibilidad social y medioambiental (Schwab, 2016; Leon, 2018; Roblek, Erenda y Meško, 2018).

- Innovación: para garantizar su supervivencia y sostenibilidad, las empresas deben mantener y desarrollar su capacidad innovadora de forma continua (Schwab, 2016).

Para alcanzar estos dos propósitos son necesarias dos capacidades generales dinámicas: la capacidad de acción sostenible y la capacidad de innovación. Y estas capacidades generales, a su vez, se deben desarrollar 
a través de un conjunto de capacidades específicas interrelacionadas:

- Flexibilidad organizacional: las empresas exitosas serán aquellas que muestren agilidad para adecuarse a nuevas circunstancias, responder a cambios imprevistos y aprovechar las nuevas oportunidades (Varanasi, 2018).

- Ambidestreza(1): la consecución del equilibrio entre exploración de nuevas oportunidades y explotación de las ventajas existentes será imprescindible (Bhandari, 2018)

- Capacidad de aprendizaje organizacional: para afrontar con éxito entornos volátiles e inciertos se requieren personas y organizaciones con grandes deseos de aprender (Lichtenthaler, 2009).

- Capacidad de gestión de conocimiento según los procesos relativos a su creación, captación, difusión, integración y aplicación; los dos primeros corresponden a la exploración; el resto corresponden a la explotación (San-Martín-Albizuri y Rodríguez-Castellanos, 2012).

- Capacidad de gestión de riesgos: que no debe desvincularse de la capacidad para detectar oportunidades de negocio. Entendemos que, en la nueva situación, la gestión de riesgos debe basarse en (Rodríguez-Castellanos, 2017):

- Sistemas de vigilancia, competitiva y tecnológica, para detectar con la mayor rapidez los riesgos y oportunidades que pueden generar los cambios en el entorno.

- Perspectiva de un mundo VUCA globalizado, con acontecimientos difícilmente previsibles, y que frecuentemente tendrán carácter sistémico.

- Más que previsión, prevención, esto es, detección de fragilidades, que permita limitar a priori la formación de riesgos; y, si el riesgo se materializa, actuar rápidamente y con agilidad para minimizar sus consecuencias.

- No anular las fuentes de oportunidad. Resulta por tanto cada vez más decisivo un nuevo tipo de "ambidestreza", al que denominaremos Ambidestreza(2), esto es, la capacidad para aprovechar oportunidades mientras se limitan los riesgos.

- Liderazgo: es preciso un nuevo estilo, de tipo transformacional (Smith y Pourdehnad, 2018), que cambie el enfoque de las normas a los fines y valores, de las reglas y procedimientos al significado, y de la jerarquía a la autonomía (Velthuijsen et al., 2017), con capacidad de aprendizaje continuo, adaptación y puesta en cuestión de modelos conceptuales y operativos previos (Schwab, 2016). Este nuevo liderazgo deberá reunir las siguientes características:

- Construcción de confianza: las personas líderes deben ser capaces de mostrar permanen- 
Covid-19, globalización, complejidad e incertidumbre: algunas reflexiones sobre gestión empresarial en tiempos de crisis y más allá

temente la autenticidad de sus deseos y acciones.

Desarrollo de la inteligencia sistémica: compartiendo el liderazgo a través de los diferentes niveles de la organización y con los stakeholders (Gray y Purdy, 2018), fomentando la autonomía y la colaboración.

- Integración de tecnología y humanismo: la persona líder deberá conocer la tecnología y saber cómo ésta puede apoyar a las personas, para que desarrollen imaginación y creatividad en beneficio del conjunto de stakeholders. A esta capacidad para integrar habilidades tecnológicas y humanísticas la denominamos Ambidestreza(3).

\section{Conclusiones}

La "crisis de la covid-19" presenta importantes diferencias con crisis anteriores, pues no tiene un origen económico ni financiero, sino en un shock externo, pero sin frágiles coyunturas económicas o financieras previas. Ahora bien, sí coincide con otras anteriores -"crisis de la globalización" o "crisis de la complejidad" acaecidas en las tres últimas décadas-, en que la complejidad debida en gran parte a la globalización ha sido un factor principal para su desencadenamiento. Pues sin las complejas interconexiones propiciadas por la globalización, no habrían sido posibles ni la rápida expansión de la propia pandemia ni sus extensos y contagiosos efectos económicos, prácticamente imposibles de prever, pero que se auguran catastróficos para ciertos sectores y países.

Un efecto esperable, junto con el desarrollo de las TIC, será la reestructuración de las cadenas de suministro para no depender de un solo proveedor, o incluso el retorno de la producción -re-localización-, para aproximarse a los consumidores, evitar costes ecológicos, o evitar dependencia externa en el suministro de ciertos activos estratégicos, como los de tipo sanitario. Algunos ven en ello una "desglobalización". Nosotros opinamos que la globalización es un fenómeno multidimensional, en el que una ralentización -o incluso un retroceso- en algunas dimensiones no afecta al conjunto del proceso. Así, la innovación tecnológica está actualmente más globalizada que en etapas anteriores, en las cuales se hallaba concentrada en un extremo de la cadena de suministro. Igualmente, la globalización financiera y la de las TIC siguen siendo evidentes.

Otra característica de esta crisis, compartida con las anteriores y también consecuencia de la complejidad, ha sido la imprevisibilidad.

Teniendo en cuenta lo anterior, entendemos que la gestión empresarial debe orientarse, por una parte, en el corto plazo a paliar los efectos inmediatos de la crisis, y por otra, con una perspectiva más amplia, a considerar cómo se debe afrontar un mundo progresivamente más complejo e incierto.

Desde una óptica de corto plazo, la actuación dependerá del lado en que 
se sitúen los problemas, si del de los clientes o del de las operaciones, o de ambos, teniendo en cuenta cuatro campos de acción estratégicos: comunicación con la clientela, operaciones, flujos de tesorería y gestión del talento humano.

Pero, más allá de la superación de la crisis, la supervivencia de la empresa para afrontar la complejidad y la incertidumbre dependerá de la medida en que consiga cumplimentar dos propósitos básicos, como son la innovación y la sostenibilidad. Y para ello deberá generar, o reforzar, una serie de capacidades, que resumiremos en tres tipos de ambidestrezas: Ambidestreza(1): capacidad para combinar "exploración", esto es, la búsqueda y creación de nuevo conocimiento, con la "explotación" del conocimiento existente; Ambidestreza(2): capacidad para combinar la detección y aprovechamiento de nuevas oportunidades con la identificación y limitación de riesgos; Ambidestreza(3): capacidad para combinar habilidades tecnológicas por un lado y humanísticas por otro.

\section{Referencias}

Adrian, T., \& Natalucci, F. (2020). COVID-19 Crisis Poses Threat to Financial Stability. IMFBlog, 14/04/2020. Recuperado a partir de https://blogs.imf.org/2020/04/14/ covid-19-crisis-poses-threat-to-financial-stability/?utm_medium=emaiI\&utm_source=govdelivery

Arellano Yanguas, J. (2020). Sociedad y política en el nuevo mundo. El Correo, 05/04/2020, p. 53. Recuperado a partir de https://blogs.deusto. es/ethics/wp-content/uploads/sites/44/2020/04/javier-arellano-sociedad-pol\%C3\%ADtica-en-el-nuevo-mundo-coronavirus.pdf

Beck, T. (2020). Finance in the times of coronavirus. En Baldwin, R. \& Weder di Mauro, B. (Eds.): Economics in the time of COVID-19 (pp.73-77). Centre for Economic Policy Research, London, UK. Recuperado a partir de https://voxeu.org/content/economics-time-covid-19

Belinchón, F. (2020a). El petróleo cotiza en negativo. Cinco Días, 20/04/2020. Recuperado a partir de https://cincodias. elpais.com/cincodias/2020/04/20/ mercados/1587365313_479009.html

Belinchón, F. (2020b). La tormenta sigue: el mayor fondo de petróleo ronda mínimos históricos. Cinco Días, 29/04/2020. Recuperado a partir de https://cincodias.elpais. com/cincodias/2020/04/28/mercados/1588089526_256775.html

Berger-Vachon, C., Gil Lafuente, A. M., Kacprzyk, J., Kondratenko, Y., Merigó Lindahl, J. M., \& Morabito, C. F. (Eds.) (2018). Complex Systems: Solutions and Challenges in Economics, Management and Engineering. Dedicated to Professor Jaime Gil Aluja. Springer, Cham, Switzerland.

Bhandari, K. R. (2018): Balancing Exploration and Exploitation Through Customer Development Model: Leveraging Industry 4.0 for Sustainable Performance. En Brunet-Thornton, R., \& Martínez, F. (Eds.): Analyzing the Impacts of Industry 4.0 in Modern Business Environments (pp. 147-160). IG| Global, Hershey, PE.

Bofinger, P., Dullien, S., Felbermayr, G., Fuest, C., Füther, M., Südekum, J., \& 
Covid-19, globalización, complejidad e incertidumbre: algunas reflexiones sobre gestión empresarial en tiempos de crisis y más allá

Weder di Mauro, B. (2020). Economic implications of the COVID-19 crisis for Germany and economic policy measures. En Baldwin, R., \& Weder di Mauro, B. (Eds.): Mitigating the COVID Economic Crisis: Act Fast and Do Whatever It Takes (pp. 167-179). Centre for Economic Policy Research, London, UK. Recuperado a partir de https://voxeu.org/ content/mitigating-covid-economic-crisis-act-fast-and-do-whatever-it-takes

Boone, L., Haugh, D. Pain, N., \& Salins, V. (2020). Tackling the fallout from COVID-19. En Baldwin, R. \& Weder di Mauro, B. (Eds.): Economics in the time of COVID-19 (pp. 37-45). Centre for Economic Policy Research, London, UK. Recuperado a partir de https://voxeu. org/content/economics-time-covid-19

Boz, E., \& Tesar, L. (2018). Investment Hollowing Out. IMF Economic Review, 66(1), 15-30. Recuperado a partir de https://link.springer.com/article/10.1057/s41308-017-0044-2

De-Goñi-Oslé, J. M., \& Rodríguez-Castellanos, A. (2018). A Model for the Management in Organizations Based on People and Knowledge: Aspects to be Considered in its Design. En Berger-Vachon, C., Gil Lafuente, A. M., Kacprzyk, J., Kondratenko, Y., Merigó Lindahl, J. M., \& Morabito, C. F. (Eds.). Complex Systems: Solutions and Challenges in Economics, Management and Engineering. Dedicated to Professor Jaime Gil Aluja (pp. 63-82). Springer, Cham, Switzerland.

Euromoney (2020a). Government finances - After the disease, the debt. Euromoney, 23/04/2020. Recuperado a partir de https://www.economist.com/ leaders/2020/04/23/after-the-disease-the-debt

Euromoney (2020b). What would Keynes do? The pandemic will leave the rich world deep in debt, and force some hard choices. Euromoney, 23/04/2020. Recuperado a partir de https://www. economist.com/briefing/2020/04/23/ the-pandemic-will-leave-the-richworld-deep-in-debt-and-force-somehard-choices

Fanjul, E. (2020). El coronavirus, ¿nuevo impulso a la desglobalización? Blog Elcano, 12/III/2020, Real Instituto Elcano. Recuperado a partir de https:// blog.realinstitutoelcano.org/el-coronavirus-nuevo-impulso-a-la-desglobalizacion/.

Fariza, I. (2020). La economía mundial se adentra en lo desconocido. El País, 15/03/2020, p. 49. Recuperado a partir de https://elpais.com/ economia/2020-03-14/la-economia-se-adentra-en-lo-desconocido. html.

Figueras, B. (2020). Guía sobre la recesión que viene: cómo afectará a sectores y empresas. Cinco Días, 23/02/2020. Recuperado a partir de https://cincodias. elpais.com/cincodias/2020/03/23/ companias/1584952094_769921.html

Fontrodona, J., \& Muller, P. (2020). Reforzar la integridad empresarial ante la crisis del Covid-19. SSRN Paper, April, 8. Recuperado a partir de https://ssrn. com/abstract=3571706. Acceso el 21/05/2020

Gil-Aluja, J., Terceño-Gómez, A., Ferrer Comalat, J.C., Merigó-Lindahl, J.M., \& Linares Mustaros, S. (Eds.) (2015). Scientific Methods for the Treatment of Uncertainty in Social Sciences. Springer, Cham, Switzerland.

Gray, B., \& Purdy, J. M. (2018). Collaborating for Our Future: Confronting Complex Problems through Multi-Stakeholder Partnerships. Oxford University Press, Oxford, UK. 
Hallward-Driemeier, M., \& Nayyar, G. (2018). Trouble in the Making? The Future of Manufacturing-Led Development. Washington, DC: The World Bank. Recuperado a partir de https://www.worldbank.org/en/topic/ competitiveness/publication/trouble-in-the-making-the-future-of-manufacturing-led-development

Innerarity, D. (2020). La complejidad de un virus. El Diario Vasco, 19/04/2020. Recuperado a partir de https://www. diariovasco.com/opinion/complejidad-virus-20200419003155-ntvo. html

Lee, P. (2020). Can banks withstand the impact of Covid-19? Euromoney, 27/03/2020. Recuperado a partir de https://www.euromoney.com/article/ b1 kxrvx42r38r6/can-banks-withstand-the-impact-of-covid-19

Leiva, J. C., \& Guillén, E. (2020). Cuadrante de análisis estratégico para daños por la crisis. Recuperado a partir de https://www.uv.es/uvempren/Cuadrante_Analisis_Estrategico.pdf

Leon, R.-D. (Ed.) (2018). Managerial strategies for business sustainability during turbulent times. IGl Global, Hershey, PE.

Lichtenthaler, U. (2009). Absorptive capacity, environmental turbulence, and the complementarity of organizational learning processes. Academy of Management Journal, 52(4), 822-846. Recuperado a partir de https://www. jstor.org/stable/40390318?seq=1

Lucchese, M., \& Pianta, M. (2020). The Coming Coronavirus Crisis: What Can We Learn? Intereconomics, 55(2), 98104. Recuperado a partir de https:// www.intereconomics.eu/contents/ year/2020/number/2/article/the-co- ming-coronavirus-crisis-what-can-welearn.html

Mack, O., Khare, A., Krämer, A., \& Burgartz, T. (Eds.) (2016). Managing in a VUCA world. Springer, Cham, Switzerland.

Mann, C. (2020). Real and financial lenses to assess the economic consequences of COVID-19. En Baldwin, R. \& Weder di Mauro, B. (Eds.): Economics in the time of COVID-19 (pp. 81-87). Centre for Economic Policy Research, London, UK. Recuperado a partir de https://voxeu.org/content/economics-time-covid-19

Marchau, V. A. W. J., Walker, W. E., Bloemen, P. J. T. M., \& Popper, S. W. (Eds.) (2019). Decision making under deep uncertainty: from theory to practice. Springer, Cham, Switzerland.

Mendoza, E.G., \& Quadrini, V. (2010). Financial globalization, financial crises and contagion. Journal of Monetary Economics, 57(1), 24-39. Recuperado a partir de https://www.sciencedirect.com/science/article/abs/pii/ S0304393209001445

Mitchell, M. (2009). Complexity, a guided tour. Oxford University Press, New York, NY.

Nolte, M. (2020). Covid-19: La economía se agrieta. El Correo, 16/03/2020. Recuperado a partir de https://www. elcorreo.com/opinion/covid19-economia-agrieta-20200316195454-nt.html

Prigogine, I., \& Stengers, I. (1984). Order out of Chaos: Man's new dialogue with nature. Bantam Books, New York, NY.

Ramelli, S., \& Wagner, A. F. (2020). Feverish Stock Price Reactions to COVID-19. Swiss Finance Institute Research Paper Series N²0-12. Recupera- 
Covid-19, globalización, complejidad e incertidumbre: algunas reflexiones sobre gestión empresarial en tiempos de crisis y más allá

do a partir de https://ssrn.com/abstract $=3550274$

Reinhart, C. M. (2002). Default, Currency Crises, and Sovereign Credit Ratings The World Bank Economic Review, 16(2), 151-170. Recuperado a partir de https://www.jstor.org/stable/3990154?seq $=1$

Reinhart, C., \& Rogoff, K. (2020). The Coronavirus DebtThreat. Wall StreetJournal, 27/03/2020. Recuperado a partir de: https://www.wsj.com/articles/the-coronavirus-debt-threat-11585262515

Roblek, V., I. Erenda, I., \& Meško, M. (2018). The Challenges of Sustainable Business Development in the Post-Industrial Society in the First Half of the 21st Century. En Leon, R.-D. (Ed.): Managerial strategies for business sustainability during turbulent times (pp. 1-22). IGI Global, Hershey, PE.

Rodríguez-Castellanos, A. (2017). ¿Un mundo más incierto? Consecuencias para la gestión de riesgos. Bilbao: Instituto de Economía Aplicada a la Empresa de la Universidad del País Vasco, 05/12/2017. Recuperado a partir de https://www.ehu.eus/ehusfera/enpresa-institutua/2017/12/05/ un-mundo-mas-incierto-consecuencias-para-la-gestion-de-riesgos/\#more-1244.

Rodríguez-Castellanos, A. (2018). La cuarta revolución industrial en las empresas: organización y personas En Las ciencias económicas y financieras ante una sociedad en transformación (pp. 27-60). Real Academia de Ciencias Económicas y Financieras, Barcelona, España. Recuperado a partir de https://racef.es/archivos/publicaciones/ racef_leon_ms_54_18.pdf

Rodríguez-Castellanos, A. (2019a). Complejidad, incertidumbre y crisis finan- ciera. En Gil-Lafuente, A. M. (Coord.): Complejidad financiera: mutabilidad $e$ incertidumbre en instituciones, mercados y productos (pp. 111-138). Real Academia de Ciencias Económicas y Financieras, Barcelona, España. Recuperado a partir de https://racef.es/ archivos/publicaciones/ms57_19_racef_mallorca_web_libro.pdf

Rodríguez-Castellanos, A. (2019b). Hacia un nuevo humanismo empresarial: organización y personas. En Un ensayo humanista para la formalización económica. Bases y aplicaciones (pp. 201-264). Real Academia de Ciencias Económicas y Financieras, Barcelona, España. Recuperado a partir de https://racef.es/archivos/publicaciones/ me58_19_web_racef_libro.pdf

Rodríguez-Castellanos, A., Urionabarrenetxea-Zabalandikoetxea, S., \& San-Martín-Albizuri, N. (2008). Crisis financieras y globalización: un análisis de sus factores determinantes. Problemas del Desarrollo, Revista Latinoamericana de Economía, 39(153), 159-183. Recuperado a partir de http://revistas.unam.mx/index.php/ pde/article/view/7714/7189

Sahagún, F. (2020). La pandemia del siglo XXI. Madri+d Notiweb, 15/04/2020. Recuperado a partir de https://www. madrimasd.org/notiweb/analisis/pandemia-siglo-xxi

San-Martín-Albizuri, N., \& Rodríguez-Castellanos, A. (2011). La imprevisibilidad de las crisis: un análisis empírico sobre los índices de riesgo país. Revista Innovar. Revista de Ciencias Administrativas y Sociales, 21(39), 163-179. Recuperado a partir de https://revistas.unal.edu.co/index.php/innovar/article/view/35093/35356

San-Martín-Albizuri, N., \& Rodríguez-Castellanos, A. (2012). Un marco concep- 
tual para los procesos de innovación abierta: integración, difusión y cooperación en el conocimiento. TELOS. Revista de Estudios Interdisciplinarios en Ciencias Sociales, 14(1), 83101. Recuperado a partir de http:// ojs.urbe.edu/index.php/telos/article/ view/1983/1875

San-Martín-Albizuri, N., \& Rodríguez-Castellanos, A. (2015). Country risk index and sovereign ratings: do they foresee financial crises? Journal of Risk Model Validation, 9(1), 33-55. Recuperado a partir de https://www.risk.net/journal-of-risk-model-validation/2400826/ country-risk-index-and-sovereign-ratings-do-they-foresee-financial-crises

Schwab, K. (2016). The Fourth Industrial Revolution. World Economic Forum, Geneva, Switzerland.

Smith, P. A. C., \& Pourdehnad, J. (2018). Organizational Leadership for the Fourth Industrial Revolution: Emerging Research and Opportunities. IGl Global, Hershey, PE. Recuperado a partir de http://www.gbv.de/dms/ zbw/1001804368.pdf
Taleb, N. N. (2007). The Black Swan: The Impact of the Highly Improbable. Random House, New York, NY.

Taleb, N. N. (2012). Antifragile: things that gain from disorder. Random House, New York, NY.

Varanasi, R. P. (2018). Enterprise Agility in Today's Era of Complexity. En Dall'Acqua, L., \& Lukose, D. (Eds.): Improving Business Performance Through Effective Managerial Training Initiatives (pp. 1-16). IGI Global, Hershey, PE.

Velthuijsen, J. W., Van Tol, W., \& Hagen, A. (2017). Human value in the digital age. PwC. Recuperado a partir de https:// www.pwc.nl/nl/assets/documents/ pwc-human-value-in-the-digital-age. pdf

Zhang, D., Hu, M., \& Ji, Q. (2020). Financial markets under the global pandemic of COVID-19. Finance Research Letters, https://doi. org/10.1016/j.frl.2020.101528. Recuperado a partir de https://www. sciencedirect.com/science/article/pii/ S1544612320304050 